\title{
Microstrip Antenna on Kapton Substrate for Strain Sensing Applications
}

\author{
Elizabeth Rufus \\ Middle East College, PC124, Knowledge Oasis Muscat, Sultanate of Oman \\ elizabeth@gmail.com
}

\begin{abstract}
In this paper the feasibility of using a circular microstrip patch antenna fabricated on flexible kapton substrate to measure strain is investigated by performing a preliminary laboratory experiment. When a patch antenna is deformed, the dimensions of the antenna changes and hence the resonant frequency of the antenna changes which is taken to be an indication of change in the strain applied. A circular microstrip patch antenna on a flexible kapton substrate operating at $4.618 \mathrm{GHz}$ has been designed based on the relation between the radius of the circular patch and its resonant frequency. The resonant frequency of the microstrip patch antenna decreases linearly with the increase in the applied strain. The shift in the resonant frequency is nearly $3 \mathrm{MHz}$ when the stain is $0.18 \%$. This microstrip circular patch antenna can be used with other components easily and can be very useful in biomedical applications and in structural health monitoring.
\end{abstract}

Keywords - Microstrip, dielectric, kapton, antenna, resonant frequency

\section{INTRODUCTION}

The recent developments in area of structural health monitoring have been tremendous and the need is constantly increasing in the area. Growth in this area has been able to develop structural health monitoring systems using various techniques. But many of the techniques are still not able to monitor conditions in a complex operational aerospace structure. Hence there is always a need to investigate on techniques and methods to develop wireless sensors which are reliable and efficient enough to be used in complex environments and applications.

As previously studied by Daliri Ali [2], the current available wireless sensors are not efficient enough to be used in structural health monitoring for aerospace structures primarily due to high cost and battery power limitations.

The focus of this paper is to make a preliminary study in the laboratory to investigate the feasibility of using a microstrip patch antenna as a sensor for stain sensing applications as it can be a wireless sensing technique. The patch can be rectangular, circular, elliptical or of any other shape. In this paper, a circular patch is considered due to the simplicity of the relationship between a single dimension of the patch and the resonant frequency. The advantages of the microstrip patch antenna lies in the fact that it is light in weight, low fabrication cost and ease of fabrication.

Analytical computation and experiments have been demonstrated by U Tata et al., [1] using a square patch antenna in which the resonant frequency of a rectangular patch antenna depends on its length and width. Instead if a circular patch antenna is used, then only the radius of the patch decides the resonant frequency, thereby making the resonant frequency depend on one variable rather than on two variables as in the case of a square patch.

Kapton is used as a substrate in the flexible circuit industry. Its long flex life and ability to withstand copper etching makes it excellent choice for these applications. It is used heavily in the electronics market as the industry moves to smaller, lighter and faster components. The use kapton substrate instead of FR4 suggested by Ali et.al in [2] for the fabrication of the circular patch antenna is proposed.

\section{DESIGN AND Simulation}

The circular patch antenna as shown in the figure 1 consists of a dielectric substrate and a circular patch printed on one side of the substrate and the ground plane coated on the other side of the substrate. The patch and the ground plane are made of copper and they form an electromagnetic resonant cavity that radiates at a specific frequency called the resonant frequency. Based on the transmission line model, the resonant frequency of a circular patch antenna with radius a, substrate thickness $h$, and relative permittivity $\varepsilon_{r}$ is given by,

$$
f_{r}=\frac{1.2412 c}{2 \pi a_{e \sqrt{\varepsilon_{r}}}}
$$

where 'c' is the velocity of light in free space and is given by

$$
c=\frac{1}{\sqrt{\mu_{0} \varepsilon_{0}}}
$$

$\mathrm{a}_{\mathrm{e}} \quad$ is the effective radius of the microstrip patch

$\varepsilon_{\mathrm{r}} \quad$ is the relative permittivity of the antenna substrate

$\mu_{0} \quad$ is the permeability of free space

$\varepsilon_{0} \quad$ is the permittivity in free space

The effective radius of the patch antenna is given as, 


$$
a_{e}=a \sqrt{\left.\left\{1+\frac{2 h}{\pi a \varepsilon_{r}} \llbracket \ln \frac{\pi a}{2 h}+1.7726\right]\right\}}
$$

Where ' $a$ ' is the radius of the patch and ' $h$ ' is the thickness of the substrate. We can write eqn (3) as

$$
a_{e}=c_{1} a
$$

where,

$$
\sqrt{c_{1}=}
$$

From eqn (5) the resonant frequency can hence be expressed as,

$$
f_{r}=\frac{c_{2}}{a_{e}}
$$

where,

$$
c_{2}=\frac{1,2412 c}{2 \pi \sqrt{\varepsilon_{r}}}
$$

Let the effective radius $a_{e}$ after applying strain be denoted as $\mathrm{a}_{\mathrm{es}}$. Then it is given by,

$$
a_{e s}=a_{e}+a_{e} \varepsilon=a_{e}(1+\varepsilon)
$$

Where, ' $\varepsilon$ ' is the strain applied. Now, let the resonant frequency after applying strain be denoted as $\mathrm{f}_{\mathrm{rs}}$.

$f_{r s}=\frac{c_{2}}{a_{e s}}=\frac{c_{2}}{a_{e}(1+\varepsilon)}$

The resonant frequency shift can be found now,

$$
\nabla f=f_{r s}-f_{r}=\frac{c_{2}}{a_{e}} \llbracket \frac{1}{1+\varepsilon}-1 \rrbracket
$$

and

$$
\frac{\nabla f}{f_{r s}}=\frac{\llbracket \frac{1}{1+\varepsilon}-1 \rrbracket}{\llbracket \frac{1}{1+\varepsilon} \rrbracket}=-\varepsilon
$$

Eqn (11) shows that there is a linear relationship between the resonant frequency shift and the strain $\varepsilon_{\mathrm{r} \text {. }}$ The negative sign indicates that, with the increase in strain the resonant frequency shifts to the left. In the analytical computation of the relationship between the resonant frequency shift and strain, the radius of the patch is considered to be uniform in all directions.

Figure 1 shows the circular patch antenna simulated using CST Studio 2010. The resonant frequency of the designed antenna is $4.392 \mathrm{GHz}$.

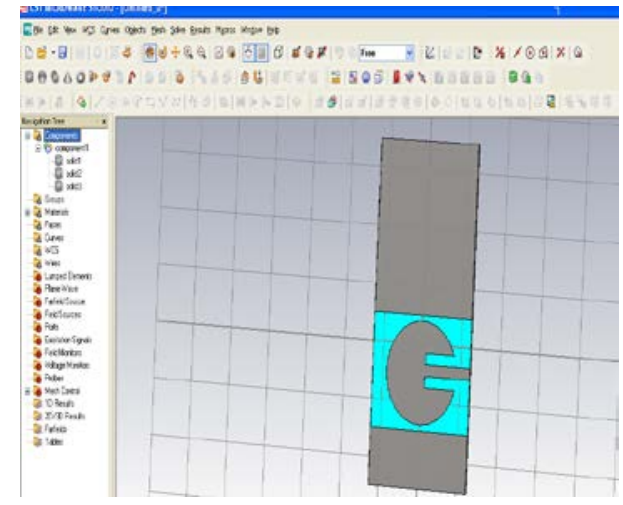

Figure 1 Circular patch antenna simulation

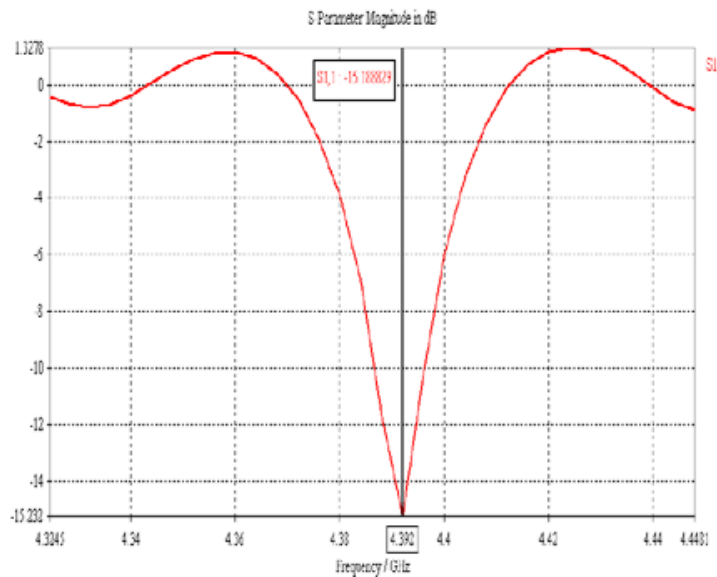

Figure 2 Return loss of the circular patch antenna

\section{III.EXPERIMENT}

The circular microstrip patch antenna was designed to operate with a resonant frequency of $4 \mathrm{GHz}$. The antenna as proposed was fabricated on a flexible kapton substrate. The antenna is fed with a microstrip line which acts as a $50 \Omega$ matched load. The fabricated antenna was bonded on an aluminum cantilever beam with its feed line perpendicular to the length of the cantilever beam. This orientation will make the patch stretch along its width when strain is applied. This changes the dimension of the patch which in turn shifts the resonant frequency of the antenna. The cantilever used in the experiment results in a strain of $0.18 \%$ on the patch when loaded with $200 \mathrm{gm}$. For the experimental purpose, the network analyzer was used as the microwave source and also for the measurement of the scattering parameter $S_{11}$. Strain was increased in steps of $0.18 \%$ and the corresponding $S_{11}$ measurements were made and plotted in the graph.

\section{IV.RESULT AND DISCUSSION}

The antenna designed was subject to different strain and the return loss is plotted below. Figure 3 shows the simulation results when the patch is strained and Figure 4 shows the experimental result when strained. The results show a clear shift in the resonant frequency.

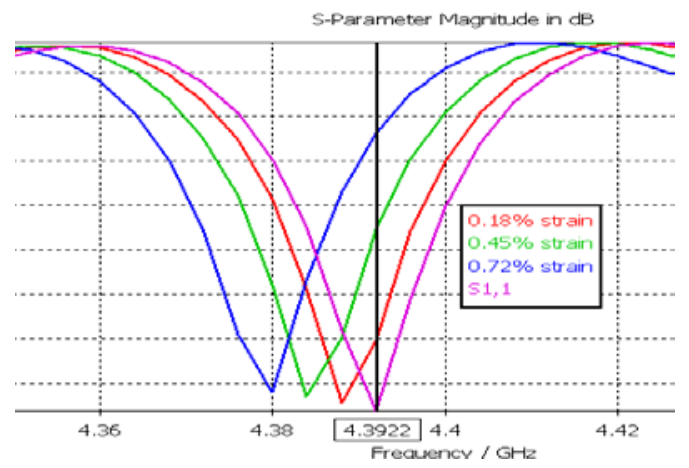

Figure 3 Circular patch antenna simulation when strained 


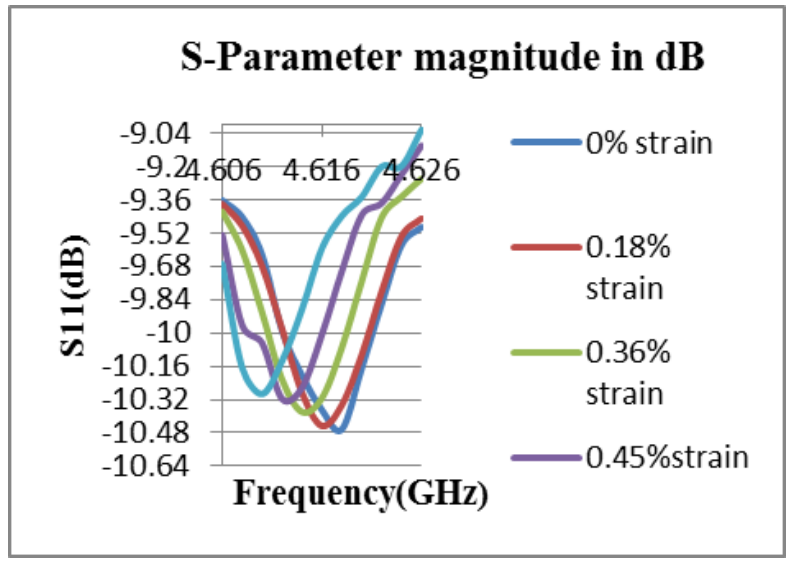

Figure 4 Experimental results of circular patch antenna when strained

TABLE 1. SIMULATION RESULTS FOR APPLIED STRAIN VERSUS RESONANT FREQUENCY

\begin{tabular}{|l|l|l|}
\hline $\begin{array}{l}\text { Load } \\
\mathrm{kg}\end{array}$ & $\begin{array}{l}\text { Strain \% } \\
\mu \text { strain }\end{array}$ & $\begin{array}{l}\text { Resonant frequency shift of } \\
\text { patch GHz }\end{array}$ \\
\hline No load & 0 & 4.392 \\
\hline 0.2 & 0.18 & 4.388 \\
\hline 0.4 & 0.36 & 4.388 \\
\hline 0.6 & 0.45 & 4.384 \\
\hline 0.8 & 0.72 & 4.380 \\
\hline
\end{tabular}

TABLE 2. EXPERIMENTAL RESULTS FOR APPLIED STRAIN VERSUS RESONANT FREQUENCY

\begin{tabular}{|l|l|l|}
\hline $\begin{array}{l}\text { Load } \\
\mathrm{kg}\end{array}$ & $\begin{array}{l}\text { Strain \% } \\
\mu \text { strain }\end{array}$ & $\begin{array}{l}\text { Resonant frequency shift of } \\
\text { patch } \quad \mathrm{GHz}\end{array}$ \\
\hline No load & 0 & 4.618 \\
\hline 0.2 & 0.18 & 4.616 \\
\hline 0.4 & 0.36 & 4.614 \\
\hline 0.6 & 0.45 & 4.612 \\
\hline 0.8 & 0.72 & 4.610 \\
\hline
\end{tabular}

This fabricated antenna when used for strain measurement also shows clear shift in the resonant frequency. The resonant frequency decreases by nearly $3 \mathrm{MHz}$ as the strain causing load increased by $0.2 \mathrm{~kg}$. The mathematical model hence derived from the simulation results and the experimental results respectively are as follows:

$$
\begin{gathered}
y=0.61 x-0.0027 \\
y=0.85 x-0.0045
\end{gathered}
$$

Where, ' $y$ ' represents the shift in the resonant frequency and ' $x$ ' represents the radius of the patch

\section{CONCLUSIONS}

The simulation and the experimental results confirm the linear relationship between the strain and the resonant frequency shift of the antenna. It is hence possible to use a circular patch microstip antenna fabricated on a kapton substrate to be used as a strain sensor in structural health monitoring applications and also effectively in biomedical applications.

\section{ACKNOWLEDGMENT}

The author would like to acknowledge the support received from the VIT University, India to carry out this work in the Microwave Laboratory.

\section{REFERENCES}

[1] U.Tata, H.Huang, R L Carter and J C Chiao, "Exploring a patch antenna for strain measurements”, Measurement Science and Technology, vol.20, IOP Publishing, 2009.

[2] Ali Daliri, Amir Galehdar, Sabu John, Wayne Rome, Kamran Ghorbani, "Circular microstrip antenna strain sensor for wireless structural health monitoring”, Proceedings of the World Congress of Engineering, vol 2. 2010.

[3] CST Microwave Studio, “Overview and work flow”, CST 1998-2010.

Dr. Elizabeth Rufus received the degree in electronics and communication engineering from Madras University, in 1991.She received the Ph.D. degree in electronics from the VIT University in 2010. Currently, she is an Assistant Professor at Middle East College, Knowledge Oasis Muscat, Oman. Her interests are in antenna design, simulation and fabrication. 